\title{
Microfabricated devices for oral drug delivery
}

Nielsen, Line Hagner; Keller, Stephan Sylvest; Boisen, Anja

Published in:
Lab on a Chip

Link to article, DOI:

$10.1039 / \mathrm{c} 8 \mathrm{lc00408k}$

Publication date:

2018

Document Version

Peer reviewed version

Link back to DTU Orbit

Citation (APA):

Nielsen, L. H., Keller, S. S., \& Boisen, A. (2018). Microfabricated devices for oral drug delivery. Lab on a Chip, 18(16), 2348-2358. https://doi.org/10.1039/c8lc00408k

- Users may download and print one copy of any publication from the public portal for the purpose of private study or research.

- You may not further distribute the material or use it for any profit-making activity or commercial gain

- You may freely distribute the URL identifying the publication in the public portal

If you believe that this document breaches copyright please contact us providing details, and we will remove access to the work immediately and investigate your claim. 


\title{
Microfabricated devices for oral drug delivery
}

Line Hagner Nielsen, Stephan Sylvest Keller, Anja Boisen*

Department of Micro- and Nanotechnology, Technical University of Denmark, Ørsteds Plads 345C, 2800 Kgs. Lyngby Denmark

*Corresponding author: Department of Micro- and Nanotechnology, Technical University of Denmark, Ørsteds Plads 345C, 2800 Kgs. Lyngby Denmark, Tel: +4545255727. Email address: anja.boisen@nanotech.dtu.dk (A. Boisen)

\begin{abstract}
Oral administration of drugs is most convenient for patients and therefore, the ultimate goal when developing new medication. The physical barriers in the body, low $\mathrm{pH}$ of the stomach and degradation by enzymes in the gastrointestinal tract are a few of the obstacles for succeeding with oral drug delivery. Microfabricated devices show promise to overcome some of these hindrances and thereby improving the bioavailability of drugs after oral administration. There is an increasing focus on microfabricated oral drug delivery systems, and so far there are three main groups of designs; patch-like structures, microcontainers and microwells. Here, we review the newest development in top-down microfabricated devices for oral drug delivery with coverage of the aspects of design, choice of material and fabrication techniques. Furthermore, the drug loading techniques and methods for testing are discussed. In addition, we discuss the future perspectives for microfabricated devices.
\end{abstract}

\section{Keywords:}

Microcontainers, patches, microwells, polymers, biopolymers, fabrication 


\section{Introduction}

Oral delivery is the preferred administration route for drugs due to its minimally invasive nature and convenience for the patients. However, it has key challenges and limitations: (i) Many potent drugs such as proteins and peptides (e.g. insulin) are unable to survive the passage through the gastrointestinal (GI) tract. They are enzymatically degraded, hydrolyzed and/or chemically deactivated in the acidic gastric environment. Further, the physical barriers in the intestine either destroy the macromolecules or prevent their absorption ${ }^{1}$. The $\mathrm{Gl}$ tract is coated by a $100 \mu \mathrm{m}$ thick mucus layer, and the drug needs to pass the mucus layer and subsequently, cross the epithelial cell layer to be absorbed in the body and distributed via the blood circulation. (ii) The release kinetics need to be controlled (time, location and amount). Some drugs need to be delivered in a 'burst mode' at a specific site, whereas others need to be released in a more controlled manner over larger parts of the GI tract. (iii) Up to $90 \%$ of the drugs in development display low solubility in the intestine and furthermore, a great portion of these have poor intestinal permeability ${ }^{2,3}$. This means that a large fraction of the drug is wasted since it is simply not dissolved in the intestinal fluids and thereby is unavailable for absorption at the intestinal wall. As a result, the oral bioavailability of the drug is compromised.

Today, oral drug dosage forms are primarily produced by powder technology and compressed into tablets. For decades, particle technologies have existed and these enable the production of drug formulation containing micro- and nanoparticulates from a variety of polymers ${ }^{4,5}$. Such formulations have, due to their spherical shape, a small contact area to the intestinal wall in comparison with other shapes such as squares or tetrahedrons ${ }^{6,7}$. Moreover, particles have the disadvantage that they may differ in size and thus, in loaded amount of drug. Lately, microdevices have been suggested as possible oral drug delivery vehicles ${ }^{8,9}$. A schematic drawing of a microfabricated drug-carrying device is shown in Figure 1, where the essential features are highlighted. It is speculated that these devices provide a unidirectional drug release since only one face of the drug reservoir can open and due to the integration of chemical or structural cues to promote oriented adhesion to the intestine wall. In this way, it might be possible to limit loss of the drug to the surroundings, as illustrated in Figure 1.

Another hypothesis is that these microfabricated devices can protect drugs until their final destination of delivery. It is anticipated that the unidirectional release as well as a sustained resident time due to mucus attachment or penetration will allow more drug to be absorbed. Indeed, the transfer of drug across an intestinal epithelial cell monolayer has been seen to increase by a factor of ten using such microdevices ${ }^{10}$. Initial animal studies have also been published ${ }^{10-12}$ showing an increase in oral bioavailability.

The concept of microfabricated devices for oral drug delivery has been discussed in several other review papers ${ }^{13-16}$. In this review, we focus on the latest developments in top-down microfabricated devices for oral drug delivery. More conventional fabrication technologies, such as microparticle realization, and larger devices such as intelligent pills or 3D printed $\mathrm{mm}$-sized tablets are not included. We will in the following cover the aspects of design, fabrication and material choice, loading of active pharmaceutical ingredients (APIs) and in vitro and in vivo testing. 


\section{Design}

The general design concept for microfabricated devices for oral drug delivery is the same: Drug is enclosed in a micro-reservoir to protect it from the environment until release of the drug is desired (often in the small intestine). However, the dimensions of the devices are different and they include more or less of the different key features highlighted in Figure 1.

The design of the microdevices is closely related to the available fabrication technologies. Also, the design has an influence on flow behavior of the device in the GI tract, adhesion or penetration through the mucus layer, amount of loaded drug and number of different drugs to be delivered simultaneously/sequentially. An overview of different realized designs is shown in Table 1 , with a classification into patches, containers and wells.

The patch-like structures have a low aspect ratio, with typical thickness of a few $\mu \mathrm{m}$ and lateral dimensions of more than $50 \mu \mathrm{m}$. Such designs have been highlighted to improve mucoadhesion because they have a low flow resistance ${ }^{17}$ and can easily contain several compartments for different drugs. Microcontainers have a higher aspect ratio, with height and diameter of similar dimensions, typically around 100-300 $\mu \mathrm{m}$. The microcontainers can in general carry a higher load of drugs and have been reported to display mucoadhesive capabilities ${ }^{11}$. To fully protect the loaded cargo, the microcontainers can be sealed with a lid of e.g. a pH-sensitive polymer to facilitate release in certain regions of the $\mathrm{Gl}$ tract ${ }^{11,12,18,19}$. More advanced designs have been proposed, where the microdevices are processed as two dimensional objects and then self-fold into container structures upon exposure to liquid ${ }^{20}$. In addition, primarily for proof-of-concept studies, microwells have been reported ${ }^{18}$. The microwells are formed as indentations in a surface and can be used to study e.g. drug loading technologies. A few examples of surface texturing of microcontainers have been published ${ }^{21}$. There, the general idea is to enhance the adhesion to the epithelial wall and potentially also to change the release profile of loaded drugs by applying e.g. hollow nanostructures on one side of microdevices.

Microdevices for oral drug delivery are still on a proof-of-concept level, and therefore the optimal design for achieving the best system promoting drug delivery and unidirectional release at the intestine wall still needs to be identified. For now, only few attempts to identify the optimal design have been made and comparative studies with various designs of microdevices are still lacking.

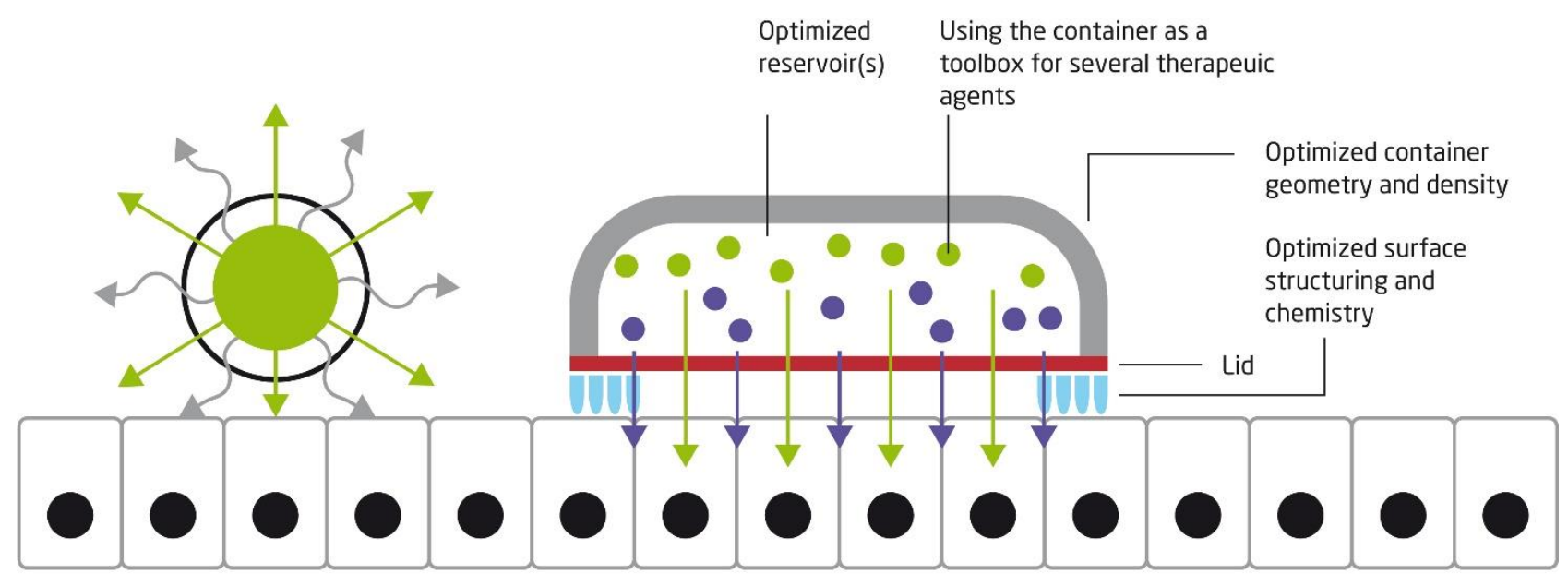

Intestinal cell membrane 
Figure 1: Left: Spherical particles release drugs in all directions, which is highly inefficient. A container structure might ensure unidirectional release of drug(s). Right: Schematic illustration of microfabricated oral drug delivery device and an indication of possible key features of the device; outer geometry, inner drug reservoir design, lid, surface structuring and surface functionalization.

Table 1: Overview of design, microfabrication technologies and materials for oral drug delivery devices

\begin{tabular}{|l|l|l|l|l|}
\hline Design & Drug delivery devices & Fabrication method & Materials & References \\
\hline & $\begin{array}{l}\text { Micropatches (single and multi- } \\
\text { compartment) }\end{array}$ & $\begin{array}{l}\text { Photolithography and } \\
\text { etching }\end{array}$ & $\mathrm{SiO}_{2}$, PMMA & $22-24$ \\
\hline & $\begin{array}{l}\text { Micropatches and } \\
\text { microcontainers }\end{array}$ & Photolithography & SU-8 & 25,26 \\
\hline & $\begin{array}{l}\text { Micropatches, self-foldable } \\
\text { microparticles }\end{array}$ & Soft lithography & PLGA, PEGDA, & 20,26 \\
\hline & Microwells & Hot embossing & PLLA, PCL & 18,27 \\
\hline & Microcontainers & Mechanical punching & PLLA & 28 \\
\hline
\end{tabular}

\section{Microfabrication technologies and materials}

The first approaches for top-down microfabrication of oral drug delivery devices have been based on traditional methods such as photolithography and etching developed by the electronics industry. An overview of the reported methods used for fabrication of the devices can be seen in Table 1 and Figure 2 . In 2001, Ahmed et al. presented initial studies on fabrication of rectangular micropatches with drug reservoirs ${ }^{23,31}$. The fabrication technology combines thin film deposition methods, photolithography and etching to define patches in $\mathrm{SiO}_{2}$ (Figure $2 \mathrm{~A}$ ). The patches have a height of a few $\mu \mathrm{m}$, lateral dimensions of 50-150 $\mu \mathrm{m}$ and contain single or multiple compartments providing volumes of 5-10 pL. A similar approach combined with electrochemical anodization has been introduced for the fabrication of porous silicon 
microparticles $^{24}$. Tao et al. have proposed the fabrication of polymer micropatches using polymethylmethacrylate (PMMA) (Figure 2A) ${ }^{22}$. This material has the advantage that it is biocompatible, already employed in many biomedical applications and suitable for surface functionalization. Photolithography combined with reactive ion etching has been used to define the patches. As a major drawback, the methods for fabrication of $\mathrm{Si}, \mathrm{SiO}_{2}$ and PMMA micropatches involve a large number of processing steps and the reservoir depth is limited to a few $\mu \mathrm{m}$.

In 2005, the same research group ${ }^{26,32}$ demonstrated fabrication of micropatches with a height of 8-12 $\mu \mathrm{m}$ using several steps of photolithography with the negative epoxy photoresist SU-8 (Figure 2B). The advantages of this method are that etching steps and secondary materials for patterning (photoresists, etch masks) can be avoided and that fabrication of devices with higher reservoir depth is relatively straightforward. For example, Nielsen et al. ${ }^{25}$ have used two steps of photolithography for the preparation of SU-8 microcontainers with lateral dimensions of $300 \mu \mathrm{m}$, a reservoir depth of $270 \mu \mathrm{m}$ and drug volumes of about $10 \mathrm{~nL}$ (Figure 2B). SU-8 is a suitable material for prototyping and initial proof-of-concept. However, the main drawback is that it is that is an epoxy based resin and potential accumulation in the GI tract could lead to unwanted side effects.

Therefore, several methods for fabrication of drug delivery devices using biodegradable polymers have been proposed ${ }^{26,27,29}$. Tao et al. have used soft lithography with SU-8 microstructures to prepare PDMS masters and subsequently performed microtransfer molding to define microwells in poly(lactic-glycolic acid) (PLGA) and gelatine (Figure $2 \mathrm{C})^{26}$. Nagstrup et al. ${ }^{27}$ have demonstrated the fabrication of microwells in biodegradable polymers poly(L-lactic acid) (PLLA) and poly(caprolactone) (PCL) by hot embossing. The stamps have been prepared by SU-8 photolithography and later replaced by electroplated $\mathrm{Ni}^{18}$. A major challenge with these traditional polymer replication methods is that a thin residual polymer layer connects the individual microwells after completed molding. This polymer film can eventually be removed by reactive ion etching or reduced by applying very high pressure during the embossing process. However, this requires either additional process steps or advanced processing equipment.

To address this issue, Petersen et al. have recently presented two approaches for fabrication of individual biodegradable polymer microcontainers based on mechanical punching (Figure $2 \mathrm{D})^{28}$ and hot punching

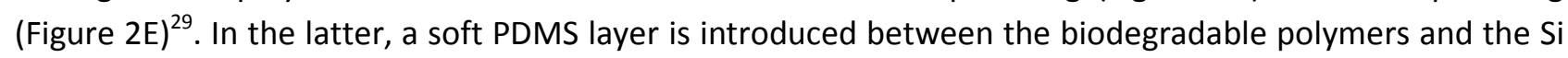
substrates. This provides the necessary elastic force during the hot embossing process to allow penetration of the thin residual layer. The microcontainers obtained with this method have a width of $300 \mu \mathrm{m}$ and a height of $100 \mu \mathrm{m}$. Alternatively, self-folding hydrogel microparticles have been fabricated and proposed as microdevices for oral drug delivery ${ }^{20}$. Soft lithography has been used for the fabrication of micropatches with lateral dimensions of 50-100 $\mu \mathrm{m}$, a height of 7-10 $\mu \mathrm{m}$ and a volume of a few pL. More specifically, resins of poly(ethylene glycol methacrylate) (PEGMA) and poly(ethylene glycol dimethacrylate) (PEGDMA) have been cast on PDMS stamps filling the stamp structures. With this approach, the residual layer typically connecting individual microdevices is avoided due to discontinuous dewetting of the PDMS mold used for casting of the polymers.

Several other methods have been introduced for fabrication of microdevices for applications in drug delivery. For example, mesoporous Si-based microparticles have been prepared by etching ${ }^{33,34}$ and monodisperse polymeric particles have been fabricated by particle replication in non-wetting templates (PRINT) $)^{35,36}$ or hydrogel templating ${ }^{37,38}$. However, although a future application of these microdevices for oral delivery can be possible, most of these studies have focused either on intravenous delivery or more fundamental aspects related to microfabrication. 
Most of the fabrication methods discussed above provide no or incomplete encapsulation of the drug. For development of a complete oral drug delivery system, the drug should be protected through the stomach. Therefore, a $\mathrm{pH}$-sensitive coating, dissolving when the microdevices reach the $\mathrm{pH}$ of the intestine (pH 6-7), is often desirable ${ }^{39}$. To date, only a few methods for microfabrication of devices for oral drug delivery with completely sealed drug reservoirs have been developed. Recently, spray coating has been introduced for the deposition of Eudragit L100 or S100 films on SU-8 and biopolymer microcontainers loaded with drug (Figure $2 \mathrm{~B})^{12,18,19}$. These polymer lids are stable in simulated gastric medium $(\mathrm{pH} 2)$ and dissolve upon immersion in simulated intestinal medium $(\mathrm{pH} \mathrm{6.5)}$, triggering release of the drug. The advantage of this method is that spray coating potentially is scalable. As a drawback, the deposition method is not selective, meaning that polymer is spray coated everywhere on the sample. This can be circumvented by the implementation of a shadow mask ${ }^{40}$.

Alternatively, several approaches of thermal bonding of polymer layers for encapsulation of drug in microfabricated devices have been demonstrated. Fox et al. have deposited membranes made of polycarbonate (PC) onto PMMA-PCL microcontainers using heat assisted bonding ${ }^{21}$. Aluminum oxide nanostraws have been integrated in the PC membranes providing nanochannels for drug loading and drug release. More recently, sealing of PLGA microcontainers with thermally bonded lids has been demonstrated with a process defined as stamped assembly of polymer layers (SEAL) (Figure 2F) ${ }^{30}$. This method allows for selective deposition of membranes on the drug delivery microdevices. However, equipment for precise alignment and bonding of lid and drug reservoirs is required.

One of the challenging aspects in top-down fabrication of drug delivery microdevices is their release from the carrier substrate and subsequent harvesting at the end of the drug loading process. Several methods involving wet chemistry for removal from the Si substrate have been proposed, such as simple delamination upon immersion in basic solution ${ }^{41}$, etching of a sacrificial layer in $\mathrm{KOH}^{23}$ or dissolution of a release layer in organic solvents ${ }^{42}$. More recently, poly(acrylic acid) (PAA) and PAA-PEG have been introduced as water soluble sacrificial layers for fabrication of SU-8 and biopolymer microcontainers ${ }^{12,29}$. Hereby, it is possible to avoid the use of aggressive etchants or solvents that potentially can affect the drug delivery devices and/or the loaded drugs. To completely prevent initiation of drug release, dry processes have been established such as simple mechanical removal of devices from fluorocarbon coated carrier substrates using razor blades ${ }^{8,11}$.

At present, SU-8 based or PMMA based microcontainers fabricated by traditional microfabrication methods are still very well suited for initial proof-of-concept studies. However, the authors believe that fabrication of these microdevices in biodegradable polymers is a necessary and significant step in the direction of bringing microdevices for oral drug delivery onto the market. There is still a long way to go in terms of fabrication, and also the first steps of incorporating drugs and depositing lids on the microdevices are very essential for this development. 
A) Photolithography and reactive ion etching $22,23,31$
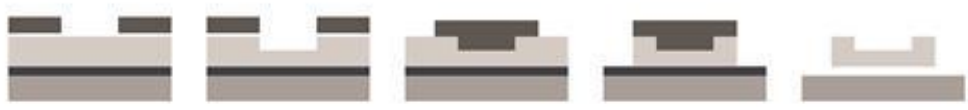

B) SU-8 photolithography and spray coating ${ }^{12,18,19,25}$
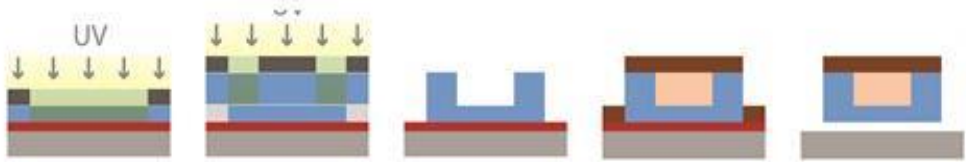

C) Soft lithography ${ }^{26}$
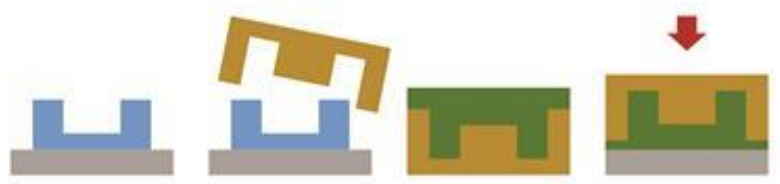

D) Hot embossing and mechanical punching ${ }^{28}$
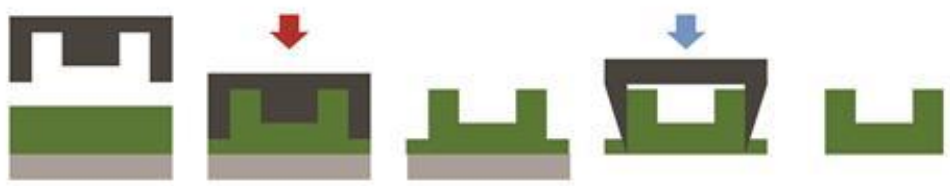

E) Hot embossing and hot punching ${ }^{29}$
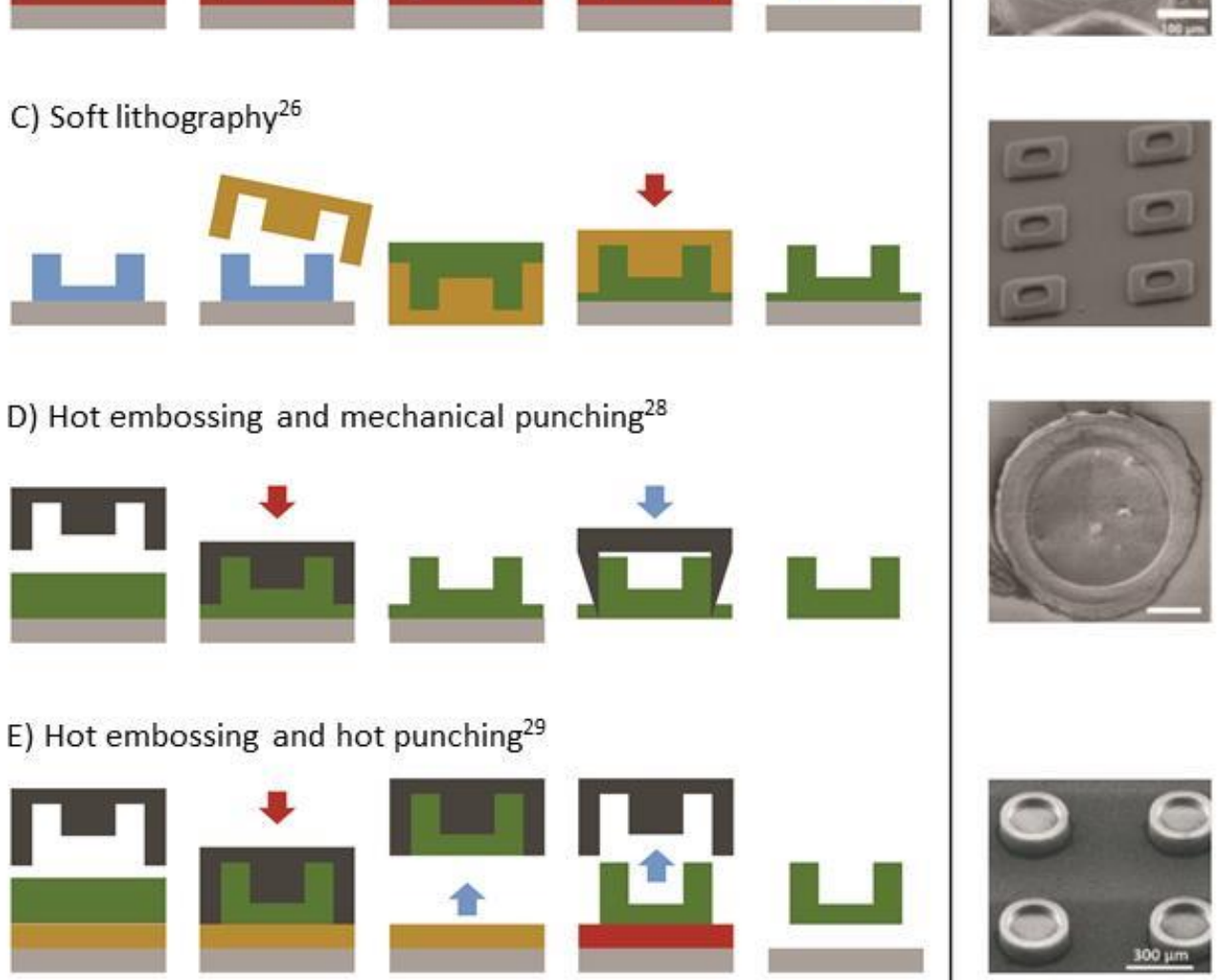

F) Stamped assembly of polymer layers $(\mathrm{SEAL})^{30}$
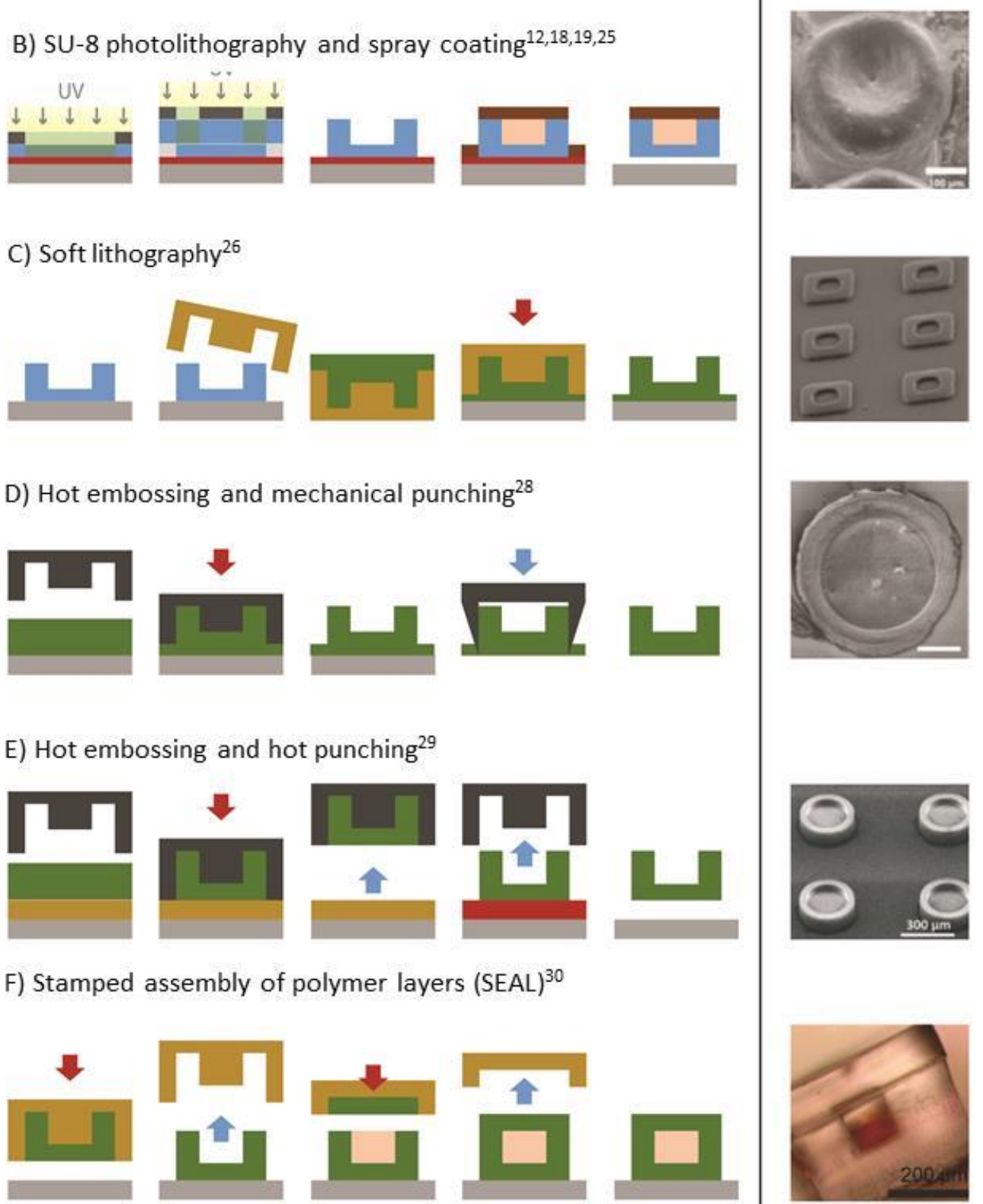

Figure 2: Schematic showing the relevant fabrication methods for drug delivery devices and with SEM images as examples of the fabricated microdevices.

\section{Loading of Active Pharmaceutical Ingredients}

After fabrication of the microdevices, it is essential to load them in an efficient way with API (Figure 3). The optimal method should allow for parallel loading of large numbers of microdevices with identical amounts of drug while providing minimal drug waste. Based on our experience, reproducible loading of API into the 
small drug reservoirs is one of the most challenging aspects in preparation of microfabricated drug delivery devices.

In initial attempts to load Si micropatches, microinjection has been used to inject biopolymer compounds dissolved in an aqueous solution into microcontainers ${ }^{31,43}$. After this, inkjet printing of solutions has been evaluated on several occasions (Figure $3 \mathrm{~A})^{31,43,44}$. Despite these attempts, the printing technique has not been established as the preferred method for direct loading of drug. The main challenges with this method are limitations on the solvents and the viscosity, which results in that the drug content in the solutions that can be printed, is relatively low. This means that multiple cycles of deposition and drying have to be performed which together with the fact that inkjet printing typically is a serial process results in a very low throughput. It could in addition also have the consequence that many more microdevices would need to be administered to the patient leading to higher costs of the medicine.

Alternatively, Marizza et al. used inkjet printing to load PVP solutions into SU-8 microcontainers. It has been possible, in a reproducible manner, to print up to $20 \mathrm{wt} \%$ polymer in aqueous solution ${ }^{44}$, directly into the microcontainers. Subsequently, the PVP has been impregnated with ketoprofen by supercritical $\mathrm{CO}_{2}$ impregnation (Figure $3 \mathrm{~B})^{45}$. This method has the advantage that organic solvents can be avoided and that drug waste can be minimized. The drawback is that the throughput of inkjet printing is low if a single nozzle is used for dispensing. Therefore, PVP has also been loaded manually as a powder and then impregnated ${ }^{12}$. This speeds up the loading process significantly. Optimization of pressure, temperature and time during impregnation resulted in a drug loading of more than $1 \mu \mathrm{g}$ in each microcontainer (Figure 3B) ${ }^{46}$. A benefit of the supercritical $\mathrm{CO}_{2}$ impregnation is that ketoprofen is converted to its amorphous form during impregnation ${ }^{12,45}$. Ketoprofen is poorly soluble in water, and therefore it is highly advantageous to have the drug in its amorphous form since this will greatly enhance solubility and dissolution rate of the drug ${ }^{47}$.

Drug-laden hydrogels have frequently been exploited for their application as an oral drug delivery system. Prepared hydrogels containing an API, e.g. fluorescein isothiocyanate (FITC)-albumin, have been spin coated into empty patches followed by selective UV photolithography to confine hydrogel areas within the reservoirs of the micropatches (Figure $3 C)^{6,17}$. A similar method has been applied with the small drug molecule, acyclovir. Here, drug-hydrogel has been loaded into micropatches with three reservoirs resulting in a load of approximately $1.5 \mathrm{ng}$ of acyclovir per reservoir (Figure $3 \mathrm{C})^{10}$. The same method has also been used to add several drug layers on top of each other inside micropatches, hereby obtaining sequential (layer-by -layer) drug release ${ }^{6,17}$.

Drug-hydrogel layers are very useful when a prolonged drug release is needed. API needs to diffuse through the hydrogel matrix, thereby delaying the release of drug from the devices. Another advantage of using UV photolithography is that large arrays of microdevices can be loaded with drug in a single step. Spin coating has the disadvantage of relatively large waste in the process. Moreover, the maximum achievable drug concentration in the hydrogel matrix can be low and UV radiation and crosslinking of the hydrogel can potentially affect the API.

In a study by Guan et al., an aqueous solution of the model drug, sodium chloride ( $\mathrm{NaCl}$ ) has been brushed into microdevices, and when the solvent evaporated, $\mathrm{NaCl}$ crystals were formed ${ }^{20}$. This method can be performed with low waste and is therefore, very beneficial when loading biopharmaceuticals into microdevices. The methods described until now have mostly been handling liquid samples, but many drugs are available in powder form and have increased stability when handled as powder, e.g. amorphous drugs 
and biopharmaceuticals. It is therefore relevant to load the microdevices with a powder. Previously, a modification of a screen printing method has been used to load microwells with an amorphous drug powder $^{18}$. The screen is applied to prevent deposition of drug powder in the area between the microwells. After alignment of the screen to the cavity of the microwells, the drug powder is distributed followed by removal of the screen. This process has resulted in filled microwells with no drug distributed between them $^{18}$. However, the alignment of such a screen is time consuming which prevents high-throughput loading. In a faster technique, powder drug has been manually loaded into microcontainers using a spatula or brush followed by the use of an airgun to remove excess drug in-between the microcontainers (Figure $3 D)^{11,25}$. This method is versatile and has also been used to load $P V P^{12}$ and drug formulation of lipid particles containing ovalbumin into microcontainers ${ }^{19}$. Typically, these methods have resulted in a minimum drug loading of $2 \mu \mathrm{g}$ per microcontainer. However, the method is time consuming, the amount of loaded drug can vary from device to device and a considerable amount of drug is wasted. An alternative loading method defined as powder embossing has been developed by Abid et al. (Figure $3 \mathrm{E}$ ) $^{48}$. Here, a metal shadow mask is aligned and clamped to the cavity of microcontainers followed by pressing the substrate into powder of either drug, polymer or lipid-based microparticles. The method has been reported to have $100 \%$ yield in terms of completely filling every microcontainer with powder and the throughput can be increased by loading drug into larger arrays of microcontainers ${ }^{48}$. Furthermore, this method has also resulted in at least $2 \mu \mathrm{g}$ of drug per microcontainer.

Alternatively, spin coating has been used to initially form a uniform drug-polymer film which is then loaded into microcontainers using hot punching (Figure $3 \mathrm{~F})^{49}$. One of the main advantages of this process is the parallel loading of large numbers of devices in a single step with the possibility to transfer it to roll-to-roll $(\mathrm{R} 2 \mathrm{R})$ processing. Furthermore, the process is versatile and avoids alignment steps. The drawbacks are that punching is performed at elevated temperatures $\left(>60^{\circ} \mathrm{C}\right)$, which might affect some drugs and that the amount of drug in spin coated drug polymer films can be relatively low due to limitations in solubility.

It can be difficult to advice on which loading method to use as it will depend greatly on the API and thereby on which dose is needed to achieve a therapeutic response in animals or humans. We believe that methods where powders are loaded into the microdevices are very versatile and are most promising for upscaling and for achieving a sufficiently high dose for testing drug-filled microdevices in animals. 


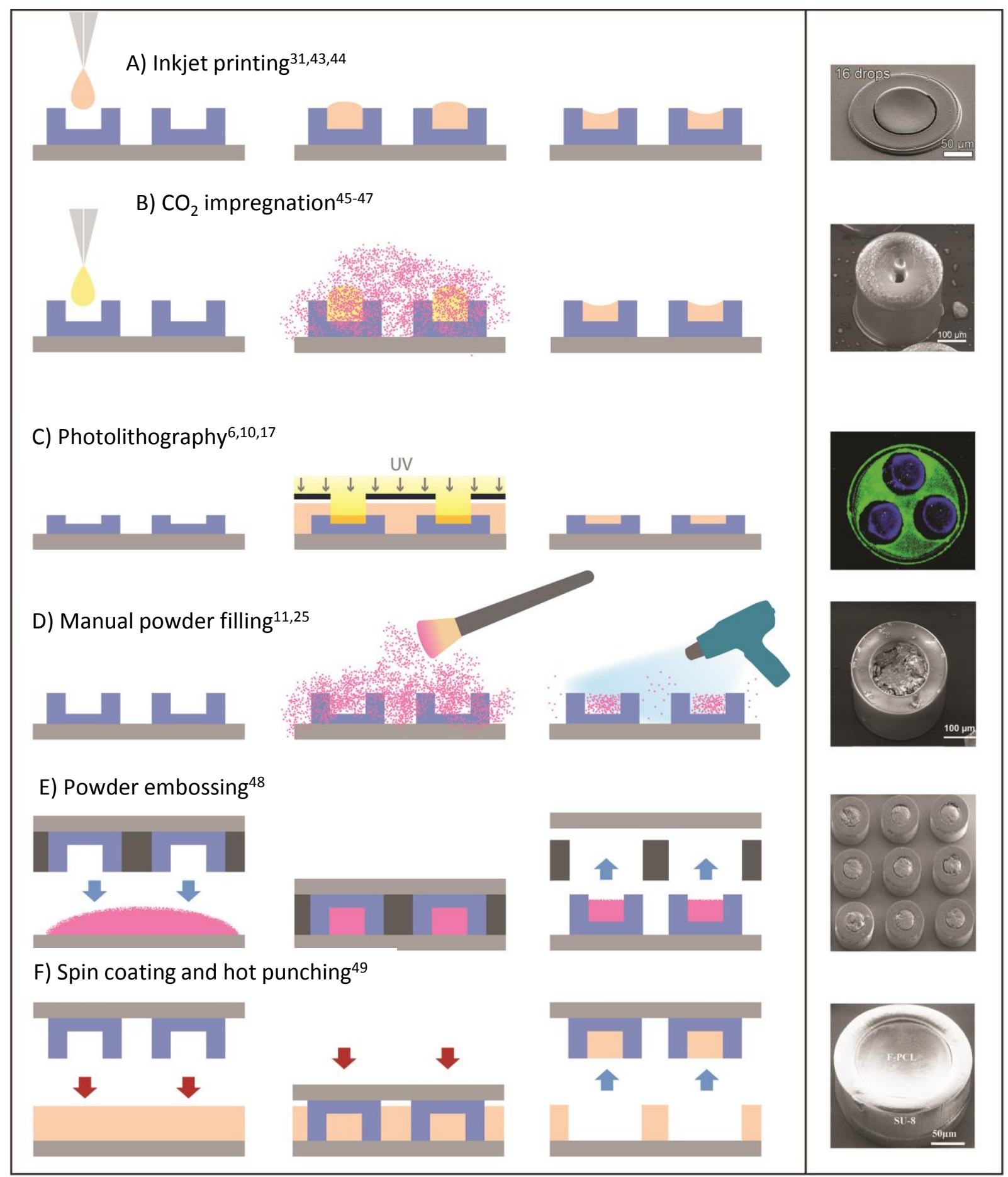

Figure 3: Graphics presenting the proposed methods for loading the fabricated microdevices with drug or drug formulation.

\section{In vitro and in vivo testing}

Fabrication of drug delivery microdevices is more time consuming and less cost efficient than e.g. producing particulates for oral drug delivery, which has been performed for decades ${ }^{50}$. To be competitive, the advantages obtained using microfabricated devices have to be considerable, and thorough in vitro and in vivo evaluation is important. In vitro studies are preferably carried out before any in vivo studies as they 
often give a good indication on how the microdevices and the API will perform. To realize a good correlation between in vitro and in vivo studies, the in vitro studies should be as close to the in vivo situation as possible. This means that media simulating the fluids in the body and animal tissue should be used for in vitro, ex vivo or in situ studies.

An overview of the methods and API's applied for testing the fabricated microdevices is shown in Table 2.

\section{Bioadhesion}

The main goal of new oral drug delivery systems is to promote the therapeutic effect of a drug, but also to minimize toxic effects. This can be done by increasing the amount of drug at the target site (usually the small intestine) to ensure rapid drug absorption and in addition, to reduce the drug concentration at nontarget sites ${ }^{51}$. The Gl transit time through the stomach and small intestine of an orally dosed device is $4-8 \mathrm{~h}$ in humans, and in many cases it is essential to prolong this time ${ }^{52}$. Therefore, one of the main aims of a delivery system is often adhesion to the GI tract and hence, mucoadhesion has been intensively studied ${ }^{51,53}$.

The Caco- 2 cell line is a commonly used intestinal cell line derived from human colonic adenocarcinoma, which is found to have many of the characteristics of the epithelium of the small intestine (tight junctions, microvilli, growth factor receptors, and major drug metabolizing enzymes) $)^{54}$. The Caco-2 cell line has become useful for investigating permeability and absorption of drugs in the development of new drug delivery systems ${ }^{54}$. It has also been widely used to test newly developed microdevices. Micropatches functionalized with tomato lectin have been incubated with Caco-2 cells to study adherence via ligandreceptor interactions ${ }^{23,31}$. It has been found that the functionalization of the micropatches resulted in improved adhesion to the Caco- 2 cells compared to non-functionalized devices. This has been explained by the flat and disc-shaped design and the small size of the micropatches ${ }^{23,31}$.

In the in vivo situation, there is a flow of liquid in the intestine. Therefore, it is important to study adhesion under flow conditions. Ainslie et al. have investigated the adhesion of micropatches functionalized with lectin to Caco-2 cells in a diffusion flow cell. By taking micrographs continuously during the study, the amount of micropatches adherent to the cells as well as the clustering effect of the microdevices have been investigated $^{8}$. It has been found that the functionalized micropatches have a significantly higher adhesion (percentage of micropatches sticking to the cells) to the cells compared to non-functionalized micropatches, $53 \%$ versus $6.8 \%$, respectively. Furthermore, the investigations have revealed that the orientation of the micropatches with respect to the flow have been random, but when the micropatches landed face down they remained attached to the Caco-2 cell monolayer during the investigated time of 30 $\mathrm{min}^{8}$. However, a major limitation of these flow studies is the fact that presence of mucus on the cells has not been demonstrated, which is not completely representing the conditions in the intestine.

For a better correlation to the in vivo situation, and as an improved investigation method, tissue from animals has been used. Gupta et al. measured the adhesive forces of $\mathrm{mm}$-sized drug-polymer patches on a piece of porcine intestinal tissue. The patches mounted on a holder and tissue have been incubated together and subsequently pulled apart. The adhesive force has been quantified using a microbalance ${ }^{55}$. A simplified method has also been used, where self-folding microdevices have been placed on a piece of inclined pig intestine. Water was added followed by optical microscopy to count the amount of maintained microdevices and to inspect the depth of engulfment in the mucosa ${ }^{20}$. Similarly, Lee et al. investigated the mucoadhesion on porcine intestinal tissue in an apparatus with a tilted angle and a continuous flow of 
simulated intestinal fluids while videos of the adhesion have been recorded ${ }^{53}$. Microcontainers have also been tested in a closed-loop in situ perfusion model in rats, where cannulas were inserted into the intestine, and through these, drug-filled microcontainers could be dosed together with fluid. After the study, the intestine has been removed and visualized to investigate how the microcontainers interacted with the intestinal membrane ${ }^{11}$.

By our experience, the best experimental method for investigating bioadhesion is a method as close to the in vivo situation as possible. If feasible, it will be advantageous to utilize tissue instead of cell lines because at present most in vitro cell models still have difficulties to represent the complex structure of intestinal tissue and the presence of mucus.

\section{In vitro drug release from the microdevices}

In vitro drug release studies can be used to evaluate efficacy of the microdevices in carrying and releasing loaded drug and to study if the microdevices provide a sustained and prolonged release. Ainslie et al. investigated if drug is released in a controlled manner from microfabricated devices. Controlled release of drug for a prolonged time is often preferred since a sudden 'burst release' of the drug can lead to unwanted side effects due to the temporarily high dose ${ }^{6}$. Furthermore, release of two drugs from the same microdevice has been investigated as a first step towards multi-drug therapy ${ }^{6}$. Drug release from the microdevices in a liquid environment is often measured by methods such as UV absorbance, fluorescence or specialized kits ${ }^{11,12,45,55,56}$. For visualization of drug release, a UV imager has previously been used. In this study, drug release from microwells has been characterized by measuring concentration and simultaneously visualizing it over time ${ }^{18}$. Raman spectroscopy has further been utilized for a qualitative measure of the drug release and to investigate the solid state form of the drug when released from the microwells ${ }^{18}$. For better correlation to the in vivo situation, media simulating the fluids in the GI tract have often been used in drug release studies ${ }^{11,12,18}$. In vitro assessment of drug release has the advantage that it is relatively fast and therefore very suited for initial investigations before performing cell studies or in vivo experiments.

\section{In vitro drug transport and toxicity}

The Caco-2 cell line is, in addition to investigations of bioadhesion, employed to study drug transport through cell layers as a measure for intestinal absorption in vivo ${ }^{54}$. In a study by Ainslie et al., fluorescein has been utilized as a model for a small molecule compound and loaded into micropatches. For these micropatches, it has been found that fluorescein has been present in high concentration at the cell interface resulting in increased drug permeability through the Caco-2 monolayer ${ }^{6}$. In another study with acyclovir, a high local drug concentration provided by the unidirectional release from the micropatches has been identified as the reason for an increased drug transport compared to controls ${ }^{10}$. Furthermore, Gupta et al. observed enhanced transport of a model protein, which they speculated to be caused by the mucoadhesiveness of the $\mathrm{mm}$-sized patches ${ }^{55}$. In another study, no significant difference has been observed in drug transport, when a drug is confined in microcontainers compared to the free drug ${ }^{11}$. This could be explained by the fact that the tested microcontainers have not been in direct contact with the cell layer but were placed in close proximity and therefore, a high local concentration of drug might not have been achieved in the same manner as in the other studies. 


\section{In vivo investigations}

Only a few animal studies on oral delivery using microfabricated devices have so far been published and a very few articles have presented specific drug delivery applications. One way to test the in vivo effect is to place the devices directly in an intestine and then measure the amount of drug absorbed in the blood ${ }^{56}$. This can be an effective method if the interaction with the intestine should be investigated in details. Gupta et al. have used this method to explore the behavior of larger patch-like drug delivery systems with different geometries ${ }^{56}$. For a better correlation to the real life situation of humans ingesting a tablet, it is important to orally dose the microdevices. This has been done in mice and rats either by oral gavage ${ }^{10,55}$ or by filling the microdevices into capsules suited for mice and rats followed by oral dosing ${ }^{11,12}$. Larger animals will often give a better correlation to humans ${ }^{51}$. To our knowledge, no testing of microdevices in larger animals has been reported so far.

SU-8 microcontainers with a diameter of approximately $300 \mu \mathrm{m}^{25}$ have proven to increase the oral bioavailability of furosemide ${ }^{11}$ in rats with $220 \%$ over a period of $24 \mathrm{~h}$ and of ketoprofen ${ }^{12}$ with $180 \%$ over $4 \mathrm{~h}$ compared to controls of the drugs not confined in microcontainers. The increased oral bioavailability is in both studies explained by the observed microcontainers penetrating into the intestinal mucus. Mazzoni et al. dosed the microcontainers orally to rats, followed by optically investigating the stomach and intestine after $90 \mathrm{~min}\left(\mathrm{~T}_{\max }\right)$ and only found microcontainers in the small intestine ${ }^{12}$.

In vivo observations are very challenging, and therefore the orientation of microdevices in the intestine has so far not been investigated in a satisfactory manner. Hence, it is still not clear if the unidirectional release provided by the microdevices is important in an in vivo situation.

Table 2: Overview of the methods and API's used when testing the microfabricated devices.

\begin{tabular}{|c|c|c|c|}
\hline Purpose & Methods & Compounds & References \\
\hline Drug release (in vitro) & $\begin{array}{l}\text { Fluorescence or UV } \\
\text { absorption }\end{array}$ & $\begin{array}{l}\text { Furosemide, ketoprofen, } \\
\text { ovalbumin, indomethacin, } \\
\text { Camptothecin, } \\
\text { Fluorescence-labelled } \\
\text { albumin, } \\
\text { insulin }\end{array}$ & $6,11,12,18,19,45,55,56,58$ \\
\hline $\begin{array}{l}\text { Displacement and } \\
\text { unidirectional release (in } \\
\text { vitro) }\end{array}$ & Caco- 2 cells with flow & Fluorescein & 8 \\
\hline $\begin{array}{l}\text { Bio- and mucoadhesion } \\
\text { (in vitro) }\end{array}$ & $\begin{array}{l}\text { Caco-2 or HT-29 cells and } \\
\text { interaction with devices }\end{array}$ & & 6,23 \\
\hline Mucoadhesion (ex vivo) & $\begin{array}{l}\text { Pig intestine with and } \\
\text { without flow }\end{array}$ & $\begin{array}{l}\text { Sodium chloride, } \\
\text { Fluorescence-labelled } \\
\text { albumin }\end{array}$ & 20,53 \\
\hline Mucoadhesion (in situ) & Intestinal perfusion in rats & Furosemide & 11 \\
\hline Drug transport (in vitro) & Caco-2 cells & $\begin{array}{l}\text { Fluorescein, furosemide, } \\
\text { acyclovir }\end{array}$ & $8,10,11$ \\
\hline Placement (in vivo) & Oral administration in rats & Ketoprofen & 12 \\
\hline
\end{tabular}




\begin{tabular}{|l|l|l|l|}
\hline $\begin{array}{l}\text { Oral bioavailability (in } \\
\text { vivo) }\end{array}$ & $\begin{array}{l}\text { Oral administration in mice } \\
\text { and rats }\end{array}$ & $\begin{array}{l}\text { Furosemide, ketoprofen, } \\
\text { acyclovir }\end{array}$ & $1^{10-12}$ \\
\hline
\end{tabular}

\section{Discussion and future perspectives}

The field of microfabricated drug delivery devices is rather new and research is still at the proof-of-concept stage. However, the past 10 years have shown considerable advances in the fabrication of micropatches, microcontainers and similar devices for oral drug delivery and their loading with drug. In the past five years, more research groups have been entering the field of microfabricated devices for oral drug delivery and focus is now also starting to be on the use of FDA approved materials. Although, initial results have been promising, it is still a major challenge to realize microdevices in biocompatible materials and to load these microdevices with large amounts of drug. Addressing this issue will in many cases mean development of new fabrication routes since these materials are rarely photosensitive. For continuous fabrication of large amounts of drug delivery devices, new fabrication routes such as roll-to roll (R2R) and embossing need to be explored - both for higher throughput and for non-lithography based processing. In particular, the challenge of highly parallel loading of microdevices with reproducible amounts of drug has to be addressed. Furthermore in the future, it is important to direct attention on toxicity of these microdevices and to load the microdevices only with excipients generally recognized as safe (GRAS).

Most of the microdevices have so far been rather simple when it comes to overall geometry and surface texturing. There are many opportunities for exploring effect of size, shape and surface texture on the flow behavior, release profiles and mucoadhesion of the microdevices. Similar studies have previously been performed on cm sized tablets fabricated using 3D printing ${ }^{60-62}$.

There are promising perspectives in microfabricated devices for oral drug delivery demonstrated both by in vitro and in vivo studies. However, to date mostly model drugs have been used for evaluation. It is essential, to identify relevant applications where major advantages are achieved compared to traditional drug delivery systems. Due to the increased fabrication costs, such applications should probably focus on therapies requiring low doses and/or where local administration in the Gl tract would be beneficial.

In terms of applications, the delivery of peptides, like insulin, is an interesting target as it could potentially help many people confronted with the inconveniences of injections, for example patients with diabetes. There is a lot of research and development in making new drug formulations for oral drug delivery of e.g. insulin ${ }^{57,59}$. Microfabricated devices offer an alternative or additional solution to challenges that oral delivery is facing. Maybe, by clever design, it is possible to deliver some of the biopharmaceuticals that can today not be delivered orally. This might not necessarily require new drug formulations but the loading of already existing drugs into miniaturized devices with new properties and new features.

Another prominent example is oral vaccination, where a microdevice would have potential to carry the vaccine formulation to release in proximity of the M-cells (uptake cells). Thereby, the immune cells in the small intestine could be activated and create immune responses. Oral vaccination would benefit children in vaccination programs both in developing and western countries. Furthermore, compliance would be improved for adults getting vaccines due to travel activities ${ }^{63}$. In addition, microdevices would be beneficial where sequential release of drugs is required for examples for HIV compounds. Here, three to four compounds often need to be administered simultaneously, and therefore it would be advantageous to 
have microdevices where the release is controlled in the order and with the timing desired to have the best effect of the drugs ${ }^{64}$.

The microdevices are significantly smaller than traditional tablets and the systems for characterization should ideally be adapted. Here, lab-on-chip devices can offer a unique platform for drug transport studies and release/degradation experiments on minimum amount of material. This has for example been demonstrated in the studies of degradation of polymers used in microcontainer fabrication ${ }^{65}$ and in the study of release from individual microcontainers. For future scenarios, it could also be interesting to integrate sensors into the drug delivery microdevices. In this way drug release could be triggered by local conditions in the Gl tract.

\section{Author contributions}

All authors contributed to the writing, discussion and correction of the review.

\section{Conflicts of interest}

The authors have no conflicts of interest to report.

\section{Acknowledgements}

The Danish National Research Foundation (Project DNRF122) and Villum Foundation's Center (Grant No. 9301) for Intelligent Drug Delivery and Sensing Using Microcontainers and Nanomechanics (IDUN) is acknowledged.

\section{References}

1 F. F. J. Martin and C. Grove, Biomed. Microdevices, 2001, 3, 97-108.

2 M. G. Fakes, B. J. Vakkalagadda, F. Qian, S. Desikan, R. B. Gandhi, C. Lai, A. Hsieh, M. K. Franchini, H. Toale and J. Brown, Int. J. Pharm., 2009, 370, 167-174.

3 L. S. Taylor and G. G. Z. Zhang, Adv. Drug Deliv. Rev., 2016, 101, 122-142.

4 L. Brannon-Peppas and J. O. Blanchette, Adv. Drug Deliv. Rev., 2012, 64, 206-212.

$5 \quad$ K. Park, J. Control. Release, 2017, 267, 2-14.

$6 \quad$ K. M. Ainslie, C. M. Kraning and T. a Desai, Lab Chip, 2008, 8, 1042-1047.

7 E. A. Klausner, E. Lavy, M. Friedman and A. Hoffman, J. Control. Release, 2003, 90, 143-162.

8 K. M. Ainslie, R. D. Lowe, T. T. Beaudette, L. Petty, E. M. Bachelder and T. A. Desai, Small, 2009, 5, 2857-2863.

9 S. Sant, S. L. Tao, O. Z. Fisher, Q. Xu, N. a. Peppas and A. Khademhosseini, Adv. Drug Deliv. Rev., 2012, 64, 496-507.

10 H. D. Chirra, L. Shao, N. Ciaccio, C. B. Fox, J. M. Wade, A. Ma and T. A. Desai, Adv. Healthc. Mater., 2014, 3, 1648-54.

11 L. H. Nielsen, A. Melero, S. S. Keller, J. Jacobsen, T. Garrigues, T. Rades, A. Müllertz and A. Boisen, Int. J. Pharm., 2016, 504, 98-109.

12 C. Mazzoni, F. Tentor, S. A. Strindberg, L. H. Nielsen, S. S. Keller, T. S. Alstrøm, C. Gundlach, A. Müllertz, P. Marizza and A. Boisen, J. Control. Release, 2017, 268, 343-351.

13 C. B. Fox, J. Kim, L. V. Le, C. L. Nemeth, H. D. Chirra and T. A. Desai, J. Control. Release, 2015, 219, 431-444.

14 H. D. Chirra and T. A. Desai, Adv. Drug Deliv. Rev., 2012, 64, 1569-1578.

15 H. J. Lee, N. Choi, E.-S. Yoon and I.-J. Cho, Adv. Drug Deliv. Rev., , DOI:10.1016/j.addr.2017.11.003. 
H. Zhang, J. K. Jackson and M. Chiao, Adv. Funct. Mater., 2017, 1703606, 1-31.

H. D. Chirra and T. A. Desai, Small, 2012, 8, 3839-3846.

L. H. Nielsen, J. Nagstrup, S. Gordon, S. S. Keller, J. Østergaard, T. Rades, A. Müllertz and A. Boisen, Biomed. Microdevices, 2015, 17, 1-7.

L. H. Nielsen, T. Rades, B. Boyd and A. Boisen, Eur. J. Pharm. Biopharm., 2017, 118, 13-20.

J. Guan, H. He, L. J. Lee and D. J. Hansford, Small, 2007, 3, 412-418.

C. B. Fox, Y. Cao, C. L. Nemeth, H. D. Chirra, R. W. Chevalier, A. M. Xu, N. A. Melosh and T. A. Desai, ACS Nano, 2016, 10, 5873-5881.

S. L. Tao, M. W. Lubeley and T. A. Desai, J. Control. Release, 2003, 88, 215-228.

A. Ahmed, C. Bonner and T. A. Desai, Biomed. Microdevices, 2001, 3, 89-96.

A. B. Foraker, R. J. Walczak, M. H. Cohen, T. A. Boiarski, C. F. Grove and P. W. Swaan, Pharm. Res., 2003, 20, 110-116.

L. H. Nielsen, S. S. Keller, K. C. Gordon, A. Boisen, T. Rades and A. Mullertz, Eur. J. Pharm. Biopharm., 2012, 81, 418-425.

S. L. Tao and T. A. Desai, Adv. Mater., 2005, 17, 1625-1629.

J. Nagstrup, S. Keller, K. Almdal and A. Boisen, Microelectron. Eng., 2011, 88, 2342-2344.

R. S. Petersen, R. Mahshid, N. K. Andersen, S. S. Keller, H. N. Hansen and A. Boisen, Microelectron. Eng., 2015, 133, 104-109.

R. S. Petersen, S. S. Keller and A. Boisen, Lab Chip, 2015, 15, $2576-9$.

K. J. McHugh, T. D. Nguyen, A. R. Linehan, D. Yang, A. M. Behrens, S. Rose, Z. L. Tochka, S. Y. Tzeng, J. J. Norman, A. C. Anselmo, X. Xu, S. Tomasic, M. A. Taylor, J. Lu, R. Guarecuco, R. Langer and A. Jaklenec, Science (80-. )., 2017, 357, 1138-1142.

A. Ahmed, C. Bonner and T. A. Desai, J. Control. Release, 2002, 81, 291-306.

S. L. Tao and T. A. Desai, Nano Lett., 2007, 7, 1463-1468.

E. Tasciotti, X. Liu, R. Bhavane, K. Plant, A. D. Leonard, B. K. Price, M. M. C. Cheng, P. Decuzzi, J. M. Tour, F. Robertson and M. Ferrari, Nat. Nanotechnol., 2008, 3, 151-157.

R. E. Serda, B. Godin, E. Blanco, C. Chiappini and M. Ferrari, Biochim. Biophys. Acta - Gen. Subj., 2011, 1810, 317-329.

J. P. Rolland, B. W. Maynor, L. E. Euliss, A. E. Exner, G. M. Denison and J. M. DeSimone, J. Am. Chem. Soc., 2005, 127, 10096-10100.

D. A. Canelas, K. P. Herlihy, K. P. Herlihy and J. M. Desimone, Wiley Interdiscip. Rev. Nanomedicine Nanobiotechnology, 2009, 1, 391-404.

G. Acharya, C. S. Shin, M. McDermott, H. Mishra, H. Park, I. C. Kwon and K. Park, J. Control. Release, 2010, 141, 314-319.

G. Acharya, C. S. Shin, K. Vedantham, M. McDermott, T. Rish, K. Hansen, Y. Fu and K. Park, J. Control. Release, 2010, 146, 201-206.

E. Sjögren, B. Abrahamsson, P. Augustijns, D. Becker, M. B. Bolger, M. Brewster, J. Brouwers, T. Flanagan, M. Harwood, C. Heinen, R. Holm, H.-P. Juretschke, M. Kubbinga, A. Lindahl, V. Lukacova, U. Münster, S. Neuhoff, M. A. Nguyen, A. Van Peer, C. Reppas, A. R. Hodjegan, C. Tannergren, W. Weitschies, C. Wilson, P. Zane, H. Lennernäs and P. Langguth, In vivo methods for drug absorption comparative physiologies, model selection, correlations with in vitro methods (IVIVC), and applications for formulation/API/excipient characterization including food effects., 2014, vol. 57. S. S. Keller, F. G. Bosco and A. Boisen, Microelectron. Eng., 2013, 110, 427-431.

S. L. Tao, M. W. Lubeley and T. A. Desai, Science (80-. )., 2003, 88, 215-228.

S. L. Tao, K. Popat and T. A. Desai, Nat. Protoc., 2007, 1, 3153-3158.

C. B. Fox, C. L. Nemeth, R. W. Chevalier, J. Cantlon, D. B. Bogdanoff, J. C. Hsiao and T. A. Desai, Bioeng. Transl. Med., 2017, 2, 9-16.

P. Marizza, S. S. Keller and A. Boisen, Microelectron. Eng., 2013, 111, 391-395.

P. Marizza, S. S. Keller, A. Müllertz and A. Boisen, J. Control. Release, 2014, 173, 1-9.

P. Marizza, L. Pontoni, T. Rindzevicius, J. F. Alopaeus, K. Su, J. A. Zeitler, S. S. Keller, I. Kikic, M. 
Moneghini, N. De Zordi, D. Solinas, A. Cortesi and A. Boisen, J. Supercrit. Fluids, 2016, 107, 145-152. H. Grohganz, P. A. Priemel, K. Lobmann, L. H. Nielsen, R. Laitinen, A. Mullertz, G. Van den Mooter and T. Rades, Expert Opin. Drug Deliv., 2014, 11, 977-989.

48 Z. Abid, C. Gundlach, O. Durucan, C. von Halling Laier, L. H. Nielsen, A. Boisen and S. S. Keller, Microelectron. Eng., 2017, 171, 20-24.

49 R. S. Petersen, S. S. Keller and A. Boisen, Macromol. Mater. Eng., 2017, 302, 1-6.

50 A. C. Anselmo and S. Mitragotri, J. Control. Release, 2014, 190, 15-28.

51 R. Malik, T. Garg, A. K. Goyal and G. Rath, J. Drug Target., 2015, 23, 109-124.

52 J. Chen, W. E. Blevins, H. Park and K. Park, J. Control. Release, 2000, 64, 39-51.

53 Y. A. L. Lee, S. Zhang, J. Lin, R. Langer and G. Traverso, Adv. Healthc. Mater., 2016, 5, 1141-1146.

54 I. J. Hildalgo, T. J. Raub and R. Borchardt, Gastroenterology, 1989, 96, 736-749.

55 V. Gupta, B. H. Hwang, N. Doshi and S. Mitragotri, J. Control. Release, 2013, 172, 541-9.

56 V. Gupta, B. H. Hwang, N. Doshi, A. Banerjee, A. C. Anselmo and S. Mitragotri, Ann. Biomed. Eng., 2016, 44, 1993-2007.

57 G. Traverso, C. M. Schoellhammer, A. Schroeder, R. Maa, G. Y. Lauwers, B. E. Polat, D. G. Anderson, D. Blankschtein and R. Langer, J. Pharm. Sci., 2015, 104, 362-367.

58 L. H. Nielsen, S. S. Keller, A. Boisen, A. Mullertz and T. Rades, Drug Deliv. Transl. Res., 2014, 4, 7.

59 A. Banerjee, J. Lee and S. Mitragotri, Bioeng. Transl. Med., 2016, 1, 338-346.

60 A. Goyanes, H. Chang, D. Sedough, G. B. Hatton, J. Wang, A. Buanz, S. Gaisford and A. W. Basit, Int. J. Pharm., 2015, 496, 414-420.

61 C. . Rowe, W. . Katstra, R. . Palazzolo, B. Giritlioglu, P. Teung and M. . Cima, J. Control. Release, 2000, 66, 11-17.

62 N. Genina, J. P. Boetker, S. Colombo, N. Harmankaya, J. Rantanen and A. Bohr, J. Control. Release, 2017, 268, 40-48.

63 J. E. Vela Ramirez, L. A. Sharpe and N. A. Peppas, Adv. Drug Deliv. Rev., 2017, 114, 116-131.

64 O. Ogbuagu, Expert Rev. Anti. Infect. Ther., 2016, 14, 1113-1126.

65 A. C. Ceccacci, C. H. Chen, E. Te Hwu, L. Morelli, S. Bose, F. G. Bosco, S. Schmid and A. Boisen, Sensors Actuators, B Chem., 2017, 241, 1303-1309. 